\title{
Interaction between hepatitis B virus infection and the efficacy of camrelizumab in combination with apatinib therapy in patients with hepatocellular carcinoma: a multicenter retrospective cohort study
}

\author{
Guosheng Yuan ${ }^{1 \#}$, Rong $\mathrm{Li}^{1 \#}$, Qi $\mathrm{Li}^{1}$, Xiaoyun $\mathrm{Hu}^{1}$, Jian Ruan ${ }^{2}$, Wenzhe Fan ${ }^{3}$, Junjie Wang ${ }^{4}$, Wei Huang ${ }^{5}$, \\ Mengya Zang ${ }^{1}$, Jinzhang Chen ${ }^{1}$
}

${ }^{1}$ Department of Infectious Diseases and Hepatology Unit, Nanfang Hospital, Southern Medical University, Guangzhou, China; ${ }^{2}$ Department of Medical Oncology, The First Affiliated Hospital, School of Medicine, Zhejiang University, Hangzhou, China; ${ }^{3}$ Department of Interventional Oncology, The First Affiliated Hospital of Sun Yat-sen University, Guangzhou, China; ${ }^{4}$ Department of Infectious Disease, The Second Affiliated Hospital of Guangzhou Medical University, Guangzhou, China; ${ }^{5}$ Department of Oncology, Shunde Hospital, Southern Medical University, Foshan, China

Contributions: (I) Conception and design: G Yuan, R Li, M Zang, J Chen; (II) Administrative support: J Chen; (III) Provision of study materials or patients: G Yuan, J Ruan, W Fan, J Wang, W Huang, M Zang; (IV) Collection and assembly of data: All authors; (V) Data analysis and interpretation: All authors; (VI) Manuscript writing: All authors; (VII) Final approval of manuscript: All authors.

\#These authors contributed equally to this work.

Correspondence to: Mengya Zang, MD; Jinzhang Chen, MD. Department of Infectious Diseases and Hepatology Unit, Nanfang Hospital, Southern Medical University, 1,838 North Guangzhou Ave., Guangzhou 510515, China. Email: zangmy@163.com; chenjinzhang@smu.edu.cn.

\begin{abstract}
Background: The interaction between hepatitis B virus (HBV) load and anti-programmed cell death (PD)-1 in combination with (+) antiangiogenic therapy remains controversial, especially for hepatocellular carcinoma (HCC) patients. This study sought to explore the effects of HBV load and antiviral therapy on anti-PD-1+ antiangiogenic therapy, and the rate of $\mathrm{HBV}$ reactivation during anti-PD-1+ antiangiogenic treatment.

Methods: We performed a multicenter retrospective cohort study of camrelizumab combined with apatinib $(\mathrm{C}+\mathrm{A})$ therapy between January 1, 2019 and January 1, 2021 in patients with unresectable HCC who were seropositive for hepatitis B surface antigen (HBsAg) and received antiviral therapy before $\mathrm{C}+\mathrm{A}$ involvement. The effects of $\mathrm{HBV}$ load and antiviral therapy on $\mathrm{C}+\mathrm{A}$ and the rate of $\mathrm{HBV}$ reactivation during $\mathrm{C}+\mathrm{A}$ treatment were examined.
\end{abstract}

Results: Eighty-six patients were included in the analysis. The patients had a mean age of 55 years, and $72(83.7 \%)$ were male. The objective response rates (ORRs) in patients with low $(<2,000 \mathrm{IU} / \mathrm{mL})$ and high $(\geq 2,000 \mathrm{IU} / \mathrm{mL})$ baseline HBV deoxyribonucleic acid (DNA) levels were $34.5 \%$ and $32.2 \%$, respectively $\left(\chi^{2}=0.046 ; \mathrm{P}=0.829\right)$, while the disease control rates (DCRs) were $67.3 \%$ and $80.6 \%$, respectively $\left(\chi^{2}=1.762\right.$; $\mathrm{P}=0.184)$. The results of the univariate and multivariate analyses showed that the baseline HBV DNA level did not affect PD. Additionally, none of the 86 patients suffered from HBV reactivation or HBV-related hepatic impairment with continuous antiviral treatment, regardless of nucleos(t)ide analogue (NA) type $(\mathrm{F}=1.473 ; \mathrm{P}=0.228)$.

Conclusions: Baseline HBV loads did not affect the tumor responses of HCC patients receiving antiPD-1+ antiangiogenic therapy. Thus, HBV reactivation should not be a contradiction for anti-PD-1+ antiangiogenic therapy among patients undergoing continuous and effective antiviral treatment.

Keywords: Hepatocellular carcinoma (HCC); hepatitis B virus (HBV); camrelizumab; apatinib; reactivation

\footnotetext{
$\wedge$ ORCID: 0000-0002-2118-4553.
} 
Submitted May 25, 2021. Accepted for publication Jun 24, 2021.

doi: 10.21037/atm-21-3020

View this article at: https://dx.doi.org/10.21037/atm-21-3020

\section{Introduction}

Hepatocellular carcinoma (HCC) ranks in the top 3 frequent causes of cancer-related mortality globally and in China $(1,2)$. Hepatitis B virus (HBV) and hepatitis C virus $(\mathrm{HCV})$ infections are the 2 main risk factors for the development of HCC worldwide, particularly in Eastern Asia and sub-Saharan Africa (3). Despite antiviral treatment being widely applied in the past decade, it has been estimated that HBV infections account for nearly $80 \%$ of HCC cases in China (4). Surgery, liver transplantation, and ablation are potentially effective strategies for the early treatment of HCC. Unfortunately, less than $30 \%$ of HCC cases can be diagnosed and treated at an early stage, which results in limited treatment options and poor prognosis (5).

For most late-stage HCC patients, the programmed cell death-1 (PD-1)/programmed cell death-ligand 1 (PD-L1) pathway inhibitor, particularly in combination with antiangiogenic therapy, have been proven to be optimal regimens for the systemic treatment of HCC (6). One study showed that the new IMbrave150 strategy was superior to that of sorafenib, and reduced the risk of death by $42 \%$. As a result, the atelizumab in combination with bevacizumab regimen became the first-line treatment for patients with advanced HCC (7). Additionally, research on the RESCUE showed that camrelizumab combined with apatinib $(\mathrm{C}+\mathrm{A})$ had a promising objective response rate (ORR) of $34.3 \%$ and a disease control rate (DCR) of $77.1 \%$ in advanced HCC patients as a first-line treatment (8). However, the above clinical trials, in which anti-PD-1/PD-L1 was used in combination with antiangiogenic therapy, excluded patients with pre-existing HBV infection or high HBV loads $(\geq 2,000 \mathrm{IU} / \mathrm{mL})$. Thus, the interaction between HBV load and anti-PD-1/PD-L1+ antiangiogenic therapy remains controversial, especially for HCC patients.

To date, few studies have reported high HBV load as a risk factor for early recurrence and/or poor overall survival (OS) after surgery in HCC patients (9-11). Zhang et al. showed that $\mathrm{HBV}$ reactivation occurs in a subset of hepatitis B surface antigen (HBsAg)-positive cancer patients undergoing anti-PD-1 or anti-PD-L1 immunotherapy (12). However, Sun et al. found that HBV loads did not compromise the clinical outcomes of HCC patients receiving anti-PD-1 inhibitors (13). To explore the effects of HBV load and antiviral therapy on anti-PD-1+ antiangiogenic therapy and the rate of $\mathrm{HBV}$ reactivation during anti-PD-1+ antiangiogenic treatment, we performed a retrospective cohort study of $\mathrm{HBV}$-associated HCC patients in China. To our knowledge, this report is novel, as it examines the interaction between $\mathrm{HBV}$ infection and anti-PD-1+ antiangiogenic therapy. We wish to share our experience with developing countries in which the majority of HCC cases are also associated with chronic HBV infection. We present the following article in accordance with the STROBE reporting checklist (available at https:// dx.doi.org/10.21037/atm-21-3020).

\section{Methods}

\section{Study design and patients}

We conducted a multicenter retrospective cohort study of C+A therapy in patients with unresectable HCC who were seropositive for HBsAg and accepted antiviral therapy before $\mathrm{C}+\mathrm{A}$ involvement. Consecutive patients referred to the following hospitals between January 1, 2019 and January 1, 2021 were enrolled in this study: (I) Nanfang Hospital, Southern Medical University; (II) the First affiliated Hospital, School of Medicine, Zhejiang University; (III) the First Affiliated Hospital of Sun Yat-sen University; (IV) the Second Affiliated Hospital of Guangzhou Medical University; and (V) Shunde Hospital, Southern Medical University. A total of 149 patients were screened for eligibility.

To be eligible to participate in this study, patients had to meet the following inclusion criteria: (I) have a pathological diagnosis of HCC; (II) be in stage $\mathrm{B} / \mathrm{C}$ according to the Barcelona Clinic Liver Cancer (BCLC) staging system (14) and be unable to tolerate or have refused surgery, ablation, radiation, or liver transplantation; (III) have received at least 2 cycles of anti-PD-1 therapy; (IV) have Child-Pugh (C-P) A or B liver function; (V) be seropositive for HBsAg and have received antiviral therapy as regular therapy before "C+A" therapy. Patients were excluded from the study if they met any of the following exclusion criteria: (I) had received apatinib or anti-PD-1 therapy previously; 


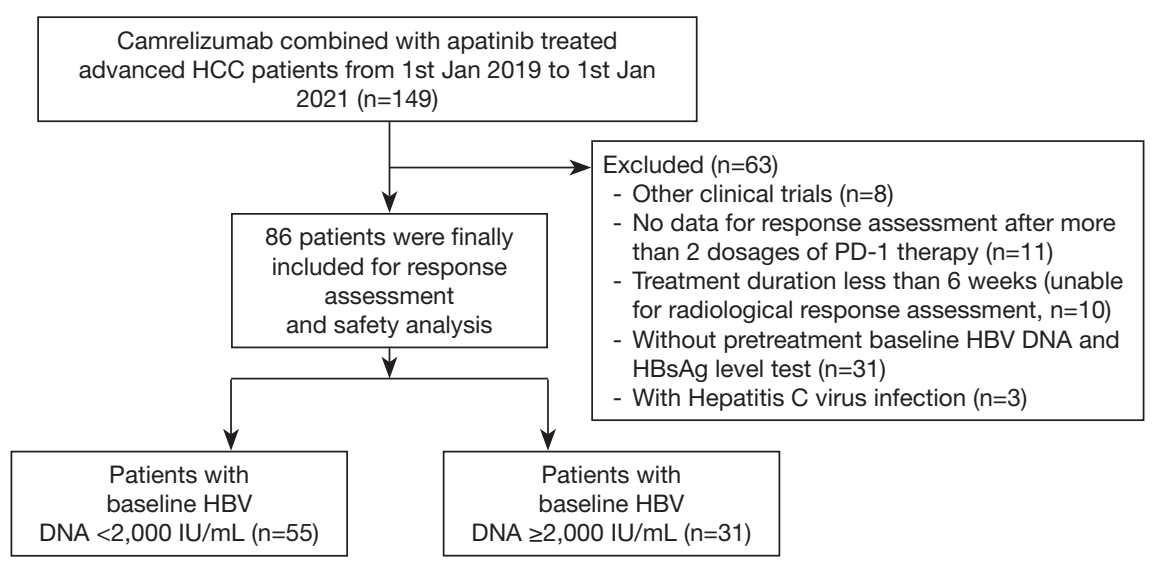

Figure 1 Flowchart of patient selection.

(II) were pregnant or breast feeding women; (III) currently had or had a history of another malignant tumor; (IV) had positive viral markers, including immunoglobulin $M$ antibodies to the hepatitis A virus, $\mathrm{HCV}$, or hepatitis $\mathrm{E}$ virus, immunoglobulin $\mathrm{G}$ antibodies to the hepatitis $\mathrm{D}$ virus or antibodies to the human immunodeficiency virus (HIV). Ultimately, 86 patients with complete data were included in this study. Figure 1 shows a flowchart of our patient selection procedure.

The reliability of this study was evaluated by calculating the power of the test based on the sample size and research outcomes. All procedures performed in this study involving human participants were in accordance with the Declaration of Helsinki (as revised in 2013). The Ethical Committee of Nanfang Hospital, Southern Medical University (NFEC2019-069) granted approval for this study, and written informed consent was obtained from each patient before the procedure.

\section{Treatment and assessment}

We described the dosage of PD-1 inhibitor and apatinib therapy in our previous study $(15,16)$. Briefly, $200 \mathrm{mg}$ of camrelizumab was administered intravenously every 3 weeks, and $250 \mathrm{mg}$ of Apatinib was administered orally daily.

Patients' demographic and clinical data were collected before $\mathrm{C}+\mathrm{A}$ therapy. Data were collected in relation to patients' age, gender, alanine aminotransferase (ALT), aspartate aminotransferase (AST), prothrombin time (PT), albumin (ALB), platelet count (PLT), total bilirubin, alphafetoprotein (AFP), BCLC stage, Eastern Cooperative Oncology Group performance, C-P score, tumor size and number, vascular invasion, and extrahepatic metastasis. An albumin-bilirubin (ALBI) score was calculated for each patient using the following formula: ALBI score $=(\log 10$ bilirubin $\times 0.66)+($ albumin $\times-0.085)$, where bilirubin is in $\mu \mathrm{mol} / \mathrm{L}$ and albumin in $\mathrm{g} / \mathrm{L}$. All patients underwent a computed tomography (CT) or magnetic resonance imaging (MRI) at the baseline, 6-12 weeks after treatment initiation, and about 3-6 months thereafter. Serological markers for $\mathrm{HBV}$ infection, including HBsAg, hepatitis B surface antibody (anti-HBs), antibody to hepatitis B core antigen anti-HBc), hepatitis $\mathrm{B}$ e-antigen ( $\mathrm{HBeAg})$, and antibody to hepatitis $\mathrm{B}$ e-antigen (anti-HBe) and $\mathrm{HBV}$ deoxyribonucleic acid (DNA), were also tested at each follow-up visit. The serum HBV DNA level was measured with the Cobras Taqman HBV Kit (Roche Diagnostics; lower limit of detection: $20 \mathrm{IU} / \mathrm{mL}$ ).

The modified response evaluation criteria in solid tumors (mRECIST) (17) were used to evaluate tumor responses. Under the mRECIST, the responses include: (I) complete response (CR): target lesions disappeared according to the enhanced imaging in the arterial phase; (II) partial response (PR): the diameter of the target lesions reduced by $\geq 30 \%$ according to the enhanced imaging in the arterial phase; (III) stable disease (SD): the diameter of the target lesions did not reduce to that in PR and did not increase to that in disease progression; (IV) progressive disease (PD): the total increase of the diameter of the target lesions (enhanced arterial phase) was $\geq 20 \%$ compared to that of the baseline value, or new lesions appeared. To reduce bias, tumor response was assessed by 2 experienced doctors with over 5 years' experience. HBV reactivation was defined according to the American Association for the Study of Liver Diseases 
2018 whereby: (I) a $\geq 2 \log$ (100-fold) increase in HBV DNA compared to the baseline level; (II) a $\geq 3,000 \mathrm{IU} / \mathrm{mL}$ in increase in HBV DNA in a patient with a previously undetectable level. HBV-related hepatitis was defined as a 3 -fold or greater increase in serum ALT level that exceeded the reference range $(58 \mathrm{U} / \mathrm{L})$ or an absolute increase in serum ALT to more than $100 \mathrm{U} / \mathrm{L}$ accompanying or following $\mathrm{HBV}$ reactivation.

\section{Statistical analysis}

The statistical analyses were performed using SPSS 20.0 software (SPSS Inc., Chicago, IL). Categorical variables are expressed as counts and percentages. In accordance with normal distribution, continuous variables are expressed as mean \pm standard deviation (SD). In accordance with nonnormal distribution, non-continuous variables are expressed as median (minimum, maximum). Qualitative differences between the subgroups were analyzed using $\chi^{2}$ tests or Fisher's exact test for categorical parameters. Survival data were calculated using the Kaplan-Meier method. Log-rank tests were used in the univariate analysis, and variables with a $\mathrm{P}$ value less than 0.1 were entered into the multivariate analysis. The multivariate analysis was performed using a Cox's proportional hazard regression model. Values of $\mathrm{P}<0.05$ were considered significant.

\section{Results}

\section{Patients}

Between January 2019 and January 2021, a total of 149 HCC patients received C+A therapy (see Figure 1). The data collection cutoff time was April 30, 2021. Sixty-three patients were excluded, as they were participating in other clinical trials $(\mathrm{n}=8)$, were co-infected with $\mathrm{HCV}(\mathrm{n}=3)$, had incomplete data $(\mathrm{n}=42)$, or had a follow-up period $<6$ weeks $(n=10)$. A total of 86 patients were included in the analysis. The power was 0.91 , which indicated a sufficient sample size and credible results.

Table 1 shows the clinical characteristics of the patients at the baseline. Patients were predominantly male $(n=72$, $83.7 \%)$, and had a mean age of 55 years ; 74.4\% (64/86) patients had BCLC C, and 50\% (43/86) had ALBI grade 1 . All patients were on antiviral prophylaxis before commencing the $\mathrm{C}+\mathrm{A}$ therapy, and the most commonly used agents were tenofovir alafenamide fumarate (TAF; $\mathrm{n}=38,44.2 \%$ ) and entecavir (ETV; $\mathrm{n}=32,37.2 \%)$. At the baseline, 55 patients $(64.0 \%)$ had a low HBV DNA level (baseline viral load $<2,000 \mathrm{IU} / \mathrm{mL}$ ), while 31 patients (36.0\%) had a high HBV DNA level (baseline viral load $\geq 2,000 \mathrm{IU} / \mathrm{mL}$ ).

\section{Tumor responses}

Tumor responses are shown in Table 2. Of all the included patients, 1 achieved a CR (1.2\%), 28 achieved a PR (32.5\%), and 33 patients had SD (27.9\%), resulting in an ORR of $33.7 \%$ and a DCR of $72.1 \%$. The subgroup analysis revealed that ORRs in patients with low and high baseline HBV DNA levels were $34.5 \%$ and $32.2 \%$, respectively $\left(\chi^{2}=0.046 ; \mathrm{P}=0.829\right)$, while the DCRs were $67.3 \%$ and $80.6 \%$, respectively $\left(\chi^{2}=1.762 ; \mathrm{P}=0.184\right)$.

\section{Correlations between the baseline variables and progressive disease}

To evaluate whether baseline variables, especially the HBV DNA level, affected PD, a logistical regression analysis was conducted. The univariate regression analysis identified the following factors as affecting PD: portal vein tumor thrombus (OR 3.336, 95\% CI, 1.374-8.005; $\mathrm{P}=0.008$ ), AFP level $\geq 400 \mathrm{ng} / \mathrm{mL}$ (OR 2.312, 95\% CI, 0.989-5.406; $\mathrm{P}=0.053)$, and ALBI grade $2 / 3(\mathrm{P}=0.003)$. We then entered these significant factors into our multivariate analysis, and found that portal vein tumor thrombus (OR 3.761, 95\% CI, 1.471-9.617; $\mathrm{P}=0.006)$ and ALBI grade $2 / 3(\mathrm{P}=0.021)$ were the only 2 independent predictive factors of PD (see Table 3 ).

\section{Effects of anti-PD-1 in combination with antiangiogenic therapy on HBV DNA and bepatitis}

During the follow-up period, none of the 86 patients suffered from HBV reactivation. As Figure 2 shows, only 3 patients suffered higher HBV DNA levels at the end of the follow-up period than at the baseline (2 received ETV, and 1 received TAF). There was no statistical difference in HBV DNA reduction among the 4 nucleos $(\mathrm{t})$ ide analogue (NA) groups $(\mathrm{F}=1.473 ; \mathrm{P}=0.228)$. Similarly, there was also no statistical difference in HBsAg reduction among the $4 \mathrm{NA}$ groups $(\mathrm{F}=0.770 ; \mathrm{P}=0.514)$. None of the treated patients achieved HBsAg or HBeAg seroclearance at the end of follow-up period (see Figure 3). Ten (11.6\%) patients experienced ALT elevation; however, all of these were considered cases of immune-related hepatitis, as no patient suffered from HBV reactivation with the continuous 
Table 1 Baseline characteristics of the 86 advanced HCC patients receiving camrelizumab in combination with apatinib therapy

\begin{tabular}{|c|c|}
\hline Characteristics & All patients $(n=86)$ \\
\hline \multicolumn{2}{|l|}{ Gender, n (\%) } \\
\hline Male & $72(83.7)$ \\
\hline Female & $14(16.3)$ \\
\hline Age $(y)^{\Delta}$ & $54.5 \pm 12.1$ \\
\hline \multicolumn{2}{|l|}{ BCLC stage, n (\%) } \\
\hline $\mathrm{B}$ & $22(25.6)$ \\
\hline C & $64(74.4)$ \\
\hline \multicolumn{2}{|l|}{ Child-Pugh class, n (\%) } \\
\hline$A$ & $64(74.4)$ \\
\hline $\mathrm{B}$ & $22(25.6)$ \\
\hline \multicolumn{2}{|l|}{ ECOG performance, n (\%) } \\
\hline 0 & $1(1.2)$ \\
\hline 1 & $54(62.8)$ \\
\hline 2 & $31(36.0)$ \\
\hline \multicolumn{2}{|c|}{ Portal vein tumor thrombus, $\mathrm{n}(\%)$} \\
\hline Yes & $40(46.5)$ \\
\hline No & $46(53.5)$ \\
\hline \multicolumn{2}{|l|}{ Extrahepatic metastasis, n (\%) } \\
\hline Yes & $20(23.3)$ \\
\hline No & $66(76.7)$ \\
\hline \multicolumn{2}{|l|}{ Tumor number, n (\%) } \\
\hline$<3$ & $39(45.3)$ \\
\hline$\geq 3$ & $47(54.7)$ \\
\hline Largest tumor diameter (cm) & $7.4(1.0,17.8)$ \\
\hline \multicolumn{2}{|l|}{$\alpha$-Fetoprotein level, $\mathrm{n}(\%)$} \\
\hline$<400 \mathrm{ng} / \mathrm{mL}$ & $42(48.8)$ \\
\hline$\geq 400 \mathrm{ng} / \mathrm{mL}$ & $44(51.2)$ \\
\hline $\operatorname{ALT}(\mathrm{U} / \mathrm{L})^{\star}$ & $30.0(5.0,216.0)$ \\
\hline AST $(U / L)^{*}$ & $42.0(9.0,231.0)$ \\
\hline Albumin $(g / L)^{\Delta}$ & $37.1 \pm 5.3$ \\
\hline Total bilirubin $(\mathrm{mmol} / \mathrm{L})^{*}$ & $13.6(5.3,78.7)$ \\
\hline $\operatorname{PLT}\left(10^{9} / \mathrm{L}\right)^{*}$ & $158.0(35.0,556.0)$ \\
\hline $\mathrm{PT}(\mathrm{s})^{*}$ & $11.6(9.7,142.0)$ \\
\hline
\end{tabular}

Table 1 (continued)
Table 1 (continued)

\begin{tabular}{|c|c|}
\hline Characteristics & All patients $(n=86)$ \\
\hline \multicolumn{2}{|c|}{ ALBI grade, n (\%) } \\
\hline 1 & $43(50.0)$ \\
\hline 2 & $37(43.0)$ \\
\hline 3 & $6(7.0)$ \\
\hline \multicolumn{2}{|l|}{ HBV DNA, n (\%) } \\
\hline$<2,000 \mathrm{IU} / \mathrm{mL}$ & $55(64.0)$ \\
\hline$\geq 2,000 \mathrm{IU} / \mathrm{mL}$ & $31(36.0)$ \\
\hline $\mathrm{HBsAg}(\mathrm{IU} / \mathrm{mL})^{*}$ & $219.8(1.71,7,379.0)$ \\
\hline \multicolumn{2}{|l|}{ HBeAg, n (\%) } \\
\hline Positive & $18(2.9)$ \\
\hline Negative & $68(79.1)$ \\
\hline \multicolumn{2}{|c|}{ Anti-viral therapy, n (\%) } \\
\hline ETV & $32(37.2)$ \\
\hline TDF & $11(12.8)$ \\
\hline TAF & $38(44.2)$ \\
\hline Others & $5(5.8)$ \\
\hline \multicolumn{2}{|c|}{$\begin{array}{l}{ }^{\triangle} \text {, normal distribution (mean } \pm \text { standard deviation); }{ }^{*} \text {, non- } \\
\text { normal distribution [median, (minimum, maximum)]. HCC, } \\
\text { hepatocellular carcinoma; BCLC, Barcelona-Clinic Liver Cancer; } \\
\text { ECOG, Eastern Cooperative Oncology Group; ALT, alanine } \\
\text { aminotransferase; AST, aspartate aminotransferase; PLT, platelet } \\
\text { count; PT, prothrombin time; others, LDV or LAM in combination } \\
\text { with ADV; ALBI, albumin-bilirubin grade = (log10 bilirubin } \times \\
0.66)+ \text { (albumin } \times-0.085 \text { ); ETV, entecavir; TDF, tenofovir; } \\
\text { TAF, tenofovir alafenamide fumarate; LDV, telbivudine; LAM, } \\
\text { lamivudine; ADV, adefovir. }\end{array}$} \\
\hline
\end{tabular}

administration of antiviral therapy.

\section{Discussion}

In the current study, we explored both the effects of HBV load and antiviral therapy on anti-PD-1+ antiangiogenic therapy and the rate of $\mathrm{HBV}$ reactivation during antiPD-1+ antiangiogenic treatment. Our findings showed that baseline HBV loads did not affect the tumor responses of anti-PD-1+ antiangiogenic treated HCC patients. However, we also proved that no HCC patient receiving continuous antiviral treatment, regardless of the type of NA, suffered from $\mathrm{HBV}$ reactivation or $\mathrm{HBV}$-related hepatitis. 
Table 2 Best tumor responses of patients with low and high baseline HBV DNA level

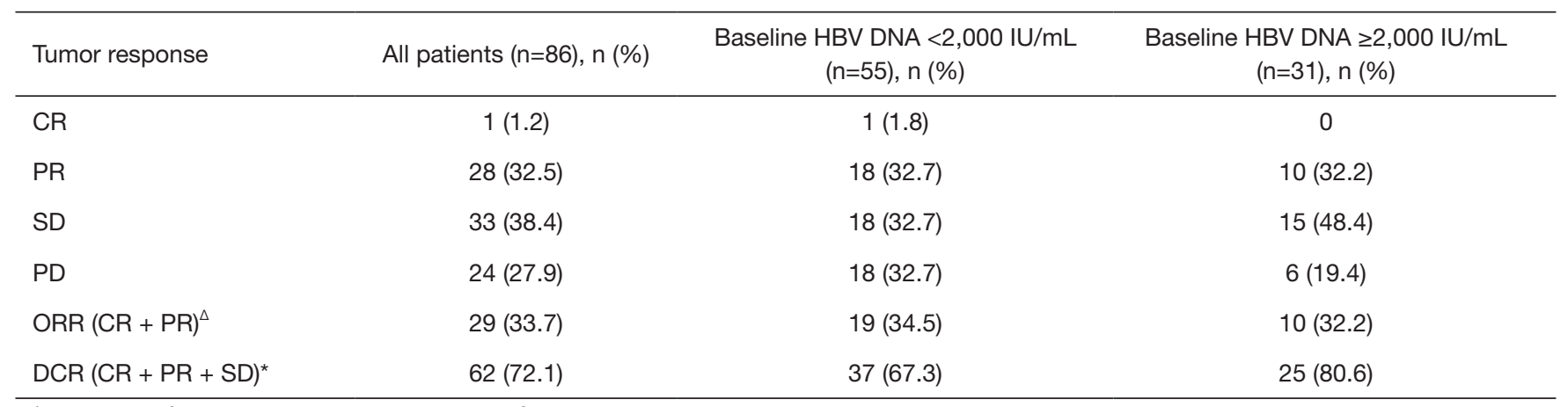

${ }^{\Delta}$, Pearson $\chi^{2}=0.046, P=0.829 ;{ }^{*}$, Pearson $\chi^{2}=1.762, P=0.184$. CR, complete response; PR, partial response; SD, stable disease; PD, progressive disease; ORR, objective response rate; $\mathrm{DCR}$, disease control rate.

As reported, viral infection could affect the clinical prognosis of anti-PD-1 therapy in gastric and anal squamous cell carcinoma $(18,19)$. It may be that viral antigens interfere with the anti-tumor effects of antiPD-1 in the tumor immune microenvironment (TME). However, a sub-study of the Acquired Immunodeficiency Syndrome Malignancy Consortium-095 Study recently reported that anti-PD-1 alone had no effect on HIV-latency or the latent HIV-reservoir (20). More importantly, Sun et al. (13) found no significant association between HBV loads and survival in HCC patients. Based on the above findings, the current results as to whether baseline viral infection interacted with antiviral treatments, especially in HCC patients, remain controversial. Unfortunately, while a substantial number of patients were $\mathrm{HBV}$-infected both in the IMbrave150 and RESCUE studies $(7,8)$, the question of whether HBV load affects the efficacy of the anti-PD-1 + antiangiogenesis regimen or the above regimen induces HBV reactivation was not assessed. Additionally, the TMEs are different between anti-PD-1 monotherapy and anti$\mathrm{PD}-1+$ antiangiogenesis combination therapy patients, as antiangiogenesis therapies could reduce vascular endothelial growth factor-mediated immunosuppression in both tumors and their TMEs and enhance the efficacy of the PD-1 inhibitor by promoting T-cell infiltration in tumors (21). The present study provided evidence that baseline HBV DNA load does not affect the tumor response of anti-PD-1 + antiangiogenesis therapy by constructing a retrospective cohort that included HCC patients who received C+A therapy. $\mathrm{HBV}$ infection may have no effect on TMEs, as it might integrate into both hepatocytes and tumor cells, and as a result, the anti-tumor ability of anti-PD-1 might not depend on HBV-associated immune attacks but on other carcinogenetic processes (22).

Another important finding of this study was that antiPD-1+ antiangiogenesis therapy had no effect on HBV reactivation and $\mathrm{HBV}$-related hepatic impairment with continuous antiviral treatment, regardless of the NA type. With the objective of "ending viral hepatitis", China has entered an era in which the application of antiviral treatments is widespread $(23,24)$. Accordingly, all the patients included in this cohort were on antiviral prophylaxis before commencing $\mathrm{C}+\mathrm{A}$ treatment, and the most commonly used agents were TAF and ETV. Consequently, we did not observe any cases of HBV reactivation or HBVrelated hepatic impairment during the follow-up period, and our incidence rates of HBV reactivation differed to those reported by Zhang et al. (12) (1.6\%) and Sun et al. (13) (1.4\%). HBV reactivation induced by anti-PD-1 might occur through the following mechanisms: (I) anti-PD-1 therapy might destroy hepatocytes and lead to the release of a previously latent virus (25); (II) the antiviral function of HBV-specific CD8+ T cells might be partially improved by the blocking of the PD-1 axis (26); or (III) the proliferation of $\mathrm{T}$ regulatory cells (Tregs) might also be promoted, leading to increased immunosuppression (27). Based on our findings, we suggest that HCC patients with positive $\mathrm{HBsAg}$ receive antiviral prophylaxis before anti-PD-1+ antiangiogenesis therapy. However, HBV reactivation must be closely monitored.

Our study had several limitations. First, this study was retrospectively designed; however, the objective endpoints (especially the imaging data for the tumor response assessments) were elaborately and integrally recorded. Second, 63 patients were excluded from this study, which may have reduced the study's statistical power; however, 
Table 3 Univariate and multivariate analysis of baseline variables affecting DCR

\begin{tabular}{|c|c|c|c|c|c|c|}
\hline Factors & \multicolumn{3}{|c|}{ Univariate } & \multicolumn{3}{|c|}{ Multivariate } \\
\hline Gender: M/F & 5.200 & $0.702-38.517$ & 0.107 & & & \\
\hline Age (y) & 1.008 & $0.974-1.043$ & 0.646 & & & \\
\hline BCLC stage: B/C & 0.561 & $0.245-1.283$ & 0.171 & & & \\
\hline ECOG performance & & & 0.500 & & & \\
\hline \multicolumn{7}{|l|}{$0^{\&}$} \\
\hline 1 & 0.000 & - & 0.979 & & & \\
\hline 2 & 0.617 & $0.277-1.378$ & 0.239 & & & \\
\hline Tumor number: $\geq 3 /<3$ & 1.088 & $0.487-2.428$ & 0.838 & & & \\
\hline Largest tumor diameter (cm) & 0.969 & $0.881-1.066$ & 0.515 & & & \\
\hline$\alpha$-Fetoprotein level: $\geq 400 /<400 \mathrm{ng} / \mathrm{mL}$ & 2.312 & $0.989-5.406$ & 0.053 & 1.944 & $0.816-4.631$ & 0.134 \\
\hline ALT (U/L) & 1.006 & $0.995-1.017$ & 0.324 & & & \\
\hline AST (U/L) & 0.996 & $0.985-1.007$ & 0.440 & & & \\
\hline Albumin (g/L) & 1.032 & $0.957-1.113$ & 0.412 & & & \\
\hline Total bilirubin (mmol/L) & 0.972 & $0.921-1.026$ & 0.309 & & & \\
\hline $\operatorname{PLT}\left(10^{9} / \mathrm{L}\right)$ & 0.998 & $0.994-1.003$ & 0.522 & & & \\
\hline Baseline HBV DNA: $\geq 2,000 /<2,000 \mathrm{IU} / \mathrm{mL}$ & 1.678 & $0.666-4.229$ & 0.272 & & & \\
\hline HBsAg (IU/mL) & 1.000 & $0.999-1.000$ & 0.119 & & & \\
\hline HBeAg: positive/negative & 3.286 & $0.772-13.983$ & 0.107 & & & \\
\hline Anti-viral therapy & & & 0.506 & & & \\
\hline \multicolumn{7}{|l|}{$\mathrm{ETV}^{\&}$} \\
\hline TDF & 0.570 & $0.125-2.603$ & 0.468 & & & \\
\hline TAF & 0.739 & $0.300-1.819$ & 0.510 & & & \\
\hline Others & 1.813 & $0.499-6.594$ & 0.366 & & & \\
\hline
\end{tabular}

\footnotetext{
\&, used as the reference category. DCR, disease control rate; BCLC, Barcelona-Clinic Liver Cancer; ECOG, Eastern Cooperative Oncology Group; ALT, alanine aminotransferase; AST, aspartate aminotransferase; PLT, platelet count; PT, prothrombin time; others, LDV or LAM in combination with ADV; ALBI, albumin-bilirubin grade $=($ log10 bilirubin $\times 0.66)+($ albumin $\times-0.085)$; ETV, entecavir; TDF, tenofovir; TAF, tenofovir alafenamide fumarate; LDV, telbivudine; LAM, lamivudine; ADV, adefovir.
} 


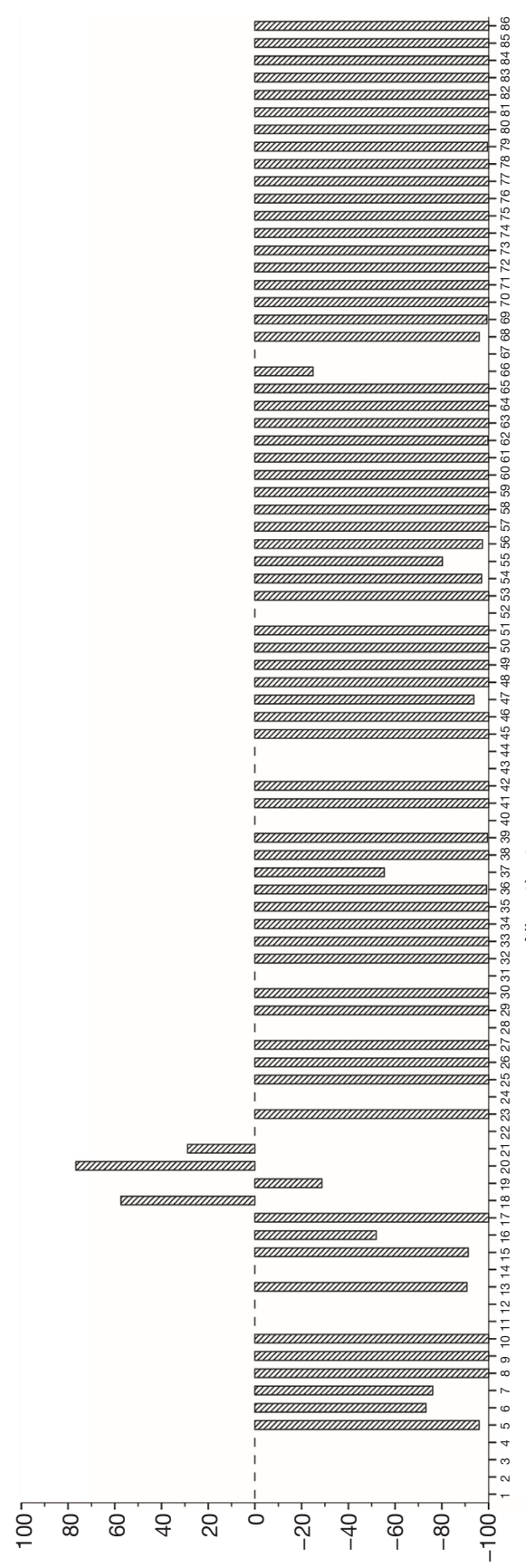

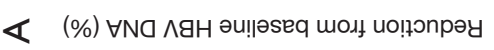

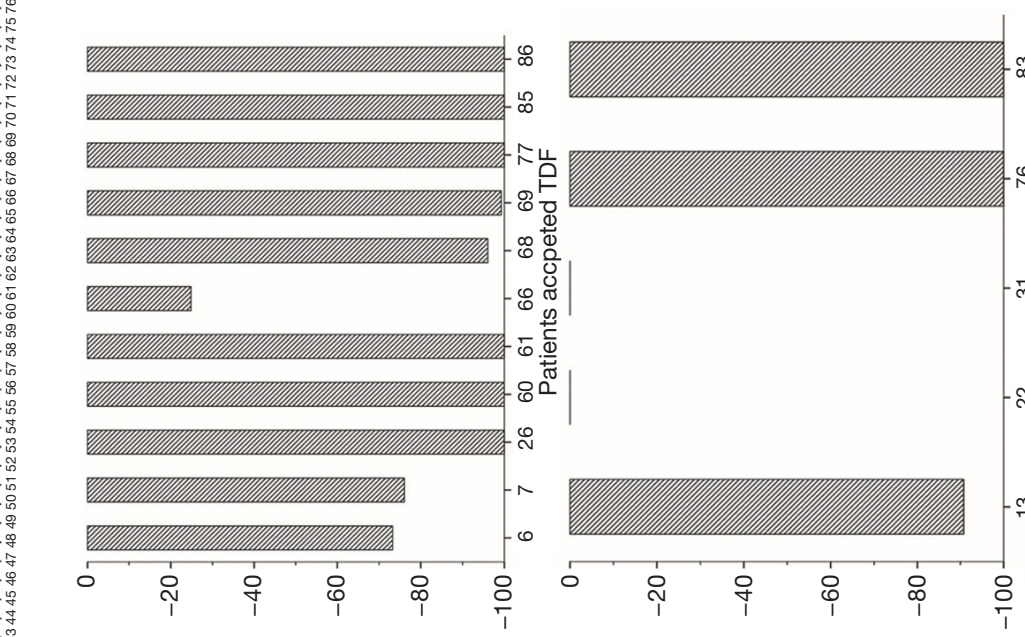

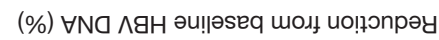
$\cup$

(\%) $\forall \mathrm{Na} \wedge$ ८H әи!|әseq moגf uo!̣эnрәу

\section{ш}

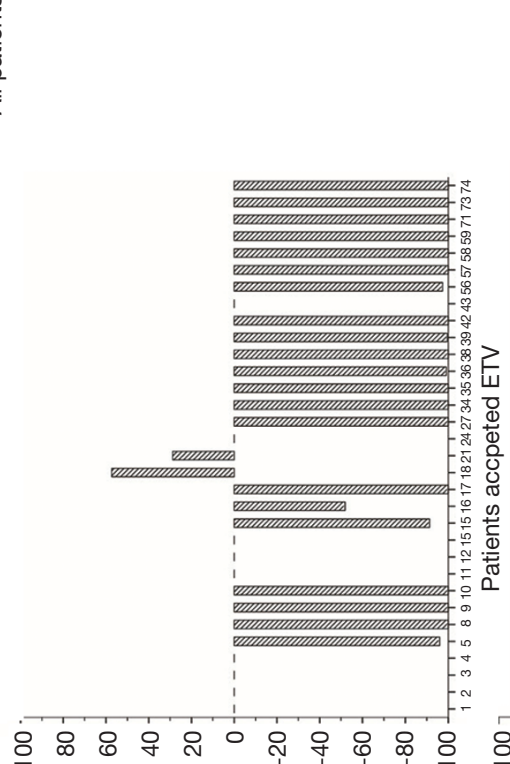

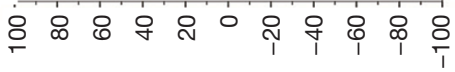

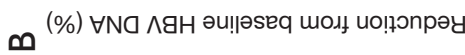

(\%) $\forall N a \wedge$ АH әи!̣әseq moג uо!̣эnрәу

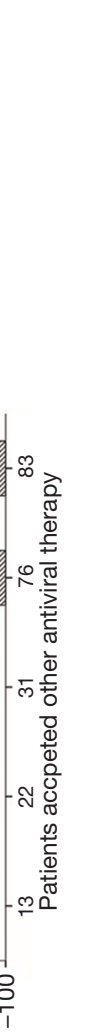




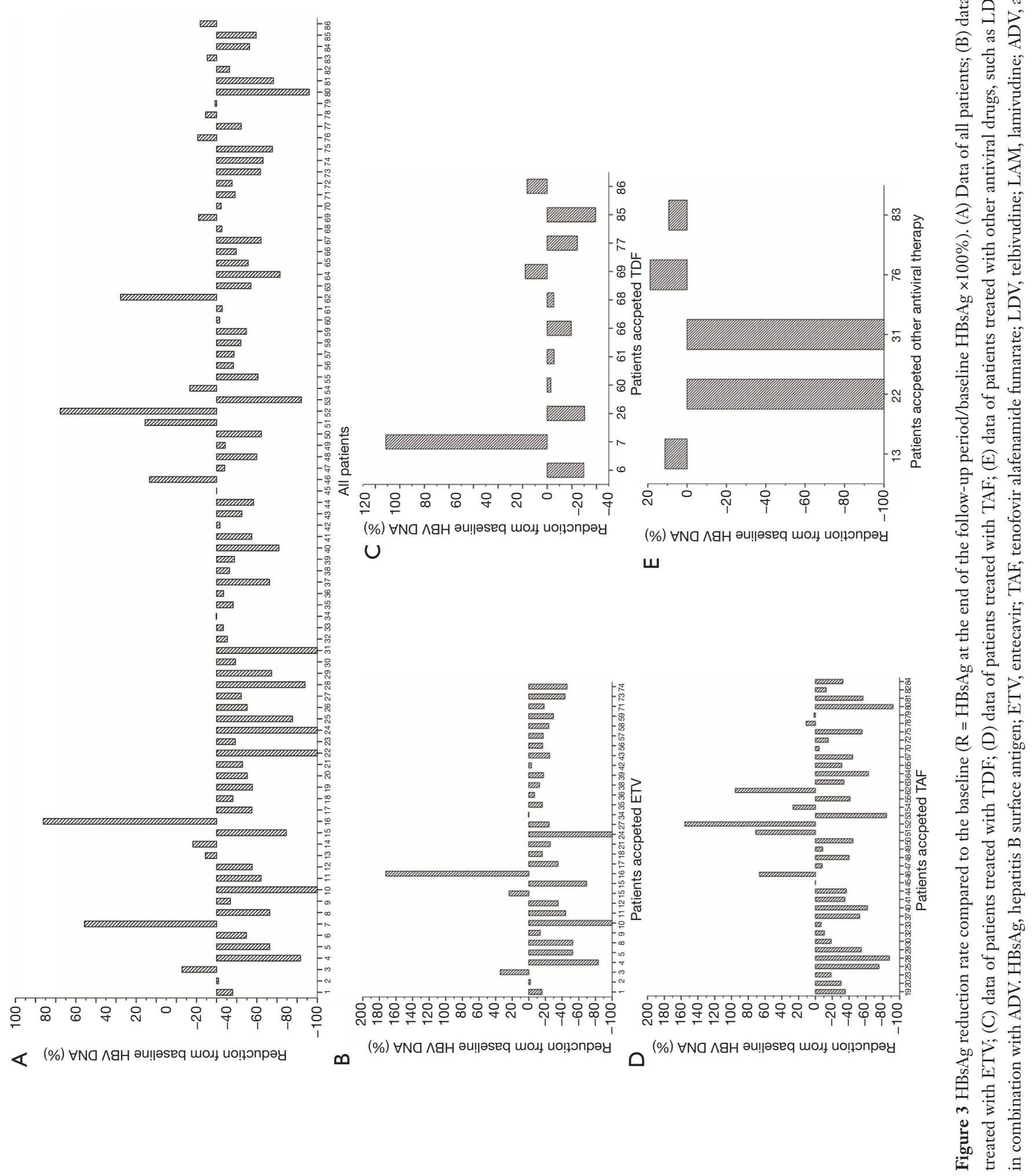


the reliability of this study was evaluated by calculating the power of the test. Third, survival data was not included in the present study, as only a fraction of the included patients died, and the follow-up period was not long enough to calculate OS. Our future research will expand the sample size and focus on the subgroup analysis of survival.

In conclusion, our findings are important, as they provide evidence that baseline HBV loads do not affect the tumor response of anti-PD-1+ antiangiogenic treated HCC patients. Further, HBV reactivation should not be a contradiction for anti-PD-1+ antiangiogenic therapy among patients undergoing continuous and effective antiviral treatment. As this study used non-randomized retrospective observational data, it only provides limited evidence that these drugs are efficacious and safe. Without further evidence-based confirmation, these data should not be taken as non-biased or used to inform clinical decisions. Future prospective studies with longer follow-up periods, larger sample sizes, and different anti-PD-1+ antiangiogenic strategies need to be conducted.

\section{Acknowledgments}

Funding: This study was partly supported by grants from the Natural Science Foundation of Guangdong Provence (2021A1515012518), the Postdoctoral Research Foundation of China (No. 2021M691468), and the Special Fund for Clinical Research of the Nanfang Hospital, Southern Medical University (2020CR019 and 2020CR021). The funding agencies had no role in the study design, data collection and analysis, decision to publish, or preparation of the manuscript.

\section{Footnote}

Reporting Checklist: The authors have completed the STROBE reporting checklist. Available at https://dx.doi. org/10.21037/atm-21-3020

Data Sharing Statement: Available at https://dx.doi. org/10.21037/atm-21-3020

Conflicts of Interest: All authors have completed the ICMJE uniform disclosure form (available at https://dx.doi. org/10.21037/atm-21-3020). The authors have no conflicts of interest to declare.

Ethical Statement: The authors are accountable for all aspects of the work in ensuring that questions related to the accuracy or integrity of any part of the work are appropriately investigated and resolved. All procedures performed in this study involving human participants were in accordance with the Declaration of Helsinki (as revised in 2013). The Ethical Committee of Nanfang Hospital, Southern Medical University (NFEC-2019-069) granted approval for this study, and written informed consent was obtained from each patient before the procedure.

Open Access Statement: This is an Open Access article distributed in accordance with the Creative Commons Attribution-NonCommercial-NoDerivs 4.0 International License (CC BY-NC-ND 4.0), which permits the noncommercial replication and distribution of the article with the strict proviso that no changes or edits are made and the original work is properly cited (including links to both the formal publication through the relevant DOI and the license). See: https://creativecommons.org/licenses/by-nc-nd/4.0/.

\section{References}

1. Xie DY, Ren ZG, Zhou J, et al. 2019 Chinese clinical guidelines for the management of hepatocellular carcinoma: updates and insights. Hepatobiliary Surg Nutr 2020;9:452-63.

2. Bray F, Ferlay J, Soerjomataram I, et al. Global cancer statistics 2018: GLOBOCAN estimates of incidence and mortality worldwide for 36 cancers in 185 countries. CA Cancer J Clin 2018;68:394-424.

3. Serio I, Napoli L, Leoni S, Piscaglia F. Direct antiviral agents for HCV infection and hepatocellular carcinoma: facts and FADs. Transl Cancer Res 2019;8:S223-32.

4. Yuen MF, Hou JL, Chutaputti A. Hepatocellular carcinoma in the Asia pacific region. J Gastroenterol Hepatol 2009;24:346-53.

5. Finn RS, Zhu AX. Evolution of Systemic Therapy for Hepatocellular Carcinoma. Hepatology 2021;73 Suppl 1:150-7.

6. Brown ZJ, Greten TF, Heinrich B. Adjuvant Treatment of Hepatocellular Carcinoma: Prospect of Immunotherapy. Hepatology 2019;70:1437-42.

7. Finn RS, Qin S, Ikeda M, et al. Atezolizumab plus Bevacizumab in Unresectable Hepatocellular Carcinoma. N Engl J Med 2020;382:1894-905.

8. $\mathrm{Xu} J$, Shen J, Gu S, et al. Camrelizumab in Combination with Apatinib in Patients with Advanced Hepatocellular Carcinoma (RESCUE): A Nonrandomized, Open-label, 
Phase II Trial. Clin Cancer Res 2021;27:1003-11.

9. Chen ZH, Zhang XP, Feng JK, et al. Actual longterm survival in hepatocellular carcinoma patients with microvascular invasion: a multicenter study from China. Hepatol Int 2021;15:642-50.

10. Chen JL, Lin XJ, Zhou Q, et al. Association of HBV DNA replication with antiviral treatment outcomes in the patients with early-stage $\mathrm{HBV}$-related hepatocellular carcinoma undergoing curative resection. Chin J Cancer 2016;35:28.

11. Liu KX, Hong JG, Wu R, et al. Clinical Benefit of Antiviral Agents for Hepatocellular Carcinoma Patients With Low Preoperative HBV-DNA Loads Undergoing Curative Resection: A Meta-Analysis. Front Oncol 2021;11:605648.

12. Zhang X, Zhou Y, Chen C, et al. Hepatitis B virus reactivation in cancer patients with positive Hepatitis B surface antigen undergoing PD-1 inhibition. J Immunother Cancer 2019;7:322.

13. Sun X, Hu D, Yang Z, et al. Baseline HBV Loads Do Not Affect the Prognosis of Patients with Hepatocellular Carcinoma Receiving Anti-Programmed Cell Death-1 Immunotherapy. J Hepatocell Carcinoma 2020;7:337-45.

14. Llovet JM, Bru C, Bruix J. Prognosis of hepatocellular carcinoma: the BCLC staging classification. Semin Liver Dis 1999; 19:329-38.

15. Chen J, Hu X, Li Q, et al. Effectiveness and safety of toripalimab, camrelizumab, and sintilimab in a realworld cohort of hepatitis B virus associated hepatocellular carcinoma patients. Ann Transl Med 2020;8:1187.

16. Yuan G, Cheng X, Li Q, et al. Safety and Efficacy of Camrelizumab Combined with Apatinib for Advanced Hepatocellular Carcinoma with Portal Vein Tumor Thrombus: A Multicenter Retrospective Study. Onco Targets Ther 2020;13:12683-93.

17. Lencioni R, Llovet JM. Modified RECIST (mRECIST) assessment for hepatocellular carcinoma. Semin Liver Dis 2010;30:52-60.

18. Chen C, Zhang F, Zhou N, et al. Efficacy and safety of immune checkpoint inhibitors in advanced gastric or gastroesophageal junction cancer: a systematic review and meta-analysis. Oncoimmunology 2019;8:e1581547.

19. Balermpas P, Martin D, Wieland U, et al. Human papilloma virus load and PD-1/PD-L1, CD8(+) and FOXP3 in anal cancer patients treated with chemoradiotherapy: Rationale for immunotherapy. Oncoimmunology 2017;6:e1288331.

20. Rasmussen TA, Rajdev L, Rhodes A, et al. Impact of antiPD-1 and anti-CTLA-4 on the HIV reservoir in people living with HIV with cancer on antiretroviral therapy: The AIDS Malignancy Consortium-095 study. Clin Infect Dis 2021. doi: 10.1093/cid/ciaa1530.

21. Deng H, Kan A, Lyu N, et al. Dual Vascular Endothelial Growth Factor Receptor and Fibroblast Growth Factor Receptor Inhibition Elicits Antitumor Immunity and Enhances Programmed Cell Death-1 Checkpoint Blockade in Hepatocellular Carcinoma. Liver Cancer 2020;9:338-57.

22. Ho WJ, Danilova L, Lim SJ, et al. Viral status, immune microenvironment and immunological response to checkpoint inhibitors in hepatocellular carcinoma. J Immunother Cancer 2020;8:e000394.

23. European Association for the Study of the Liver. European Association for the Study of the Liver. EASL Clinical Practice Guidelines: Management of hepatocellular carcinoma. J Hepatol 2018;69:182-236.

24. Heimbach JK, Kulik LM, Finn RS, et al. AASLD guidelines for the treatment of hepatocellular carcinoma. Hepatology 2018;67:358-80.

25. Hoogeveen RC, Robidoux MP, Schwarz T, et al. Phenotype and function of $\mathrm{HBV}$-specific T cells is determined by the targeted epitope in addition to the stage of infection. Gut 2019;68:893-904.

26. Lim CJ, Lee YH, Pan L, et al. Multidimensional analyses reveal distinct immune microenvironment in hepatitis B virus-related hepatocellular carcinoma. Gut 2019;68:916-27.

27. Trehanpati N, Vyas AK. Immune Regulation by T Regulatory Cells in Hepatitis B Virus-Related Inflammation and Cancer. Scand J Immunol 2017;85:175-81.

Cite this article as: Yuan G, Li R, Li Q, Hu X, Ruan J, Fan W, Wang J, Huang W, Zang M, Chen J. Interaction between hepatitis $B$ virus infection and the efficacy of camrelizumab in combination with apatinib therapy in patients with hepatocellular carcinoma: a multicenter retrospective cohort study. Ann Transl Med 2021;9(18):1412. doi: 10.21037/ atm-21-3020 